\title{
Determination of Pseudorange Error Models and Multipath Characterization under Signal-Degraded Scenarios
}

\author{
Daniel Medina $^{\dagger}$, Kasia Gibson ${ }^{\dagger *}$, Ralf Ziebold ${ }^{\dagger}$, Pau Closas* \\ ${ }^{\dagger}$ Institute of Communications and Navigation, German Aerospace Center (DLR), Germany \\ ${ }^{*}$ Dept. of Electrical \& Computer Engineering, Northeastern University, Boston MA, USA
}

\section{AUTHOR Biographies}

Daniel Medina is a research fellow at German Aerospace Center (DLR), in the Department of Nautical Systems of the Institute of Communications and Navigation. He received the B.Eng in Electrical Engineering from the University of Malaga and the M.Eng in Computer Science from Charles III University of Madrid. Currently, he is pursuing his PhD on sensor fusion schemes for precise positioning and attitude determination.

Kasia Gibson is currently pursuing a Bachelor of Science in Electrical Engineering at Northeastern University, and is expected to graduate in 2019. She worked as an intern for the Institute of Communications and Navigation at DLR, Fall of 2017.

Ralf Ziebold is a research fellow at the German Aerospace Center (DLR), in the Department of Nautical Systems of the Institute of Communications and Navigation. He received his PhD in Physics at the Goettingen University in 2000. From 20002009 he worked as a development engineer for semiconductor manufacturing at Infineon / Qimonda. Currently, he leads the group of Multi Sensor Systems. His current research topics are provision of resilient PNT data for maritime applications and integrity provision by multi sensor fusion techniques.

Pau Closas is an Assistant Professor at Northeastern University, Boston, MA. He received the MS and PhD degrees in Electrical Engineering from UPC in 2003 and 2009. He also holds a MS in Advanced Mathematics from UPC, 2014. His primary areas of interest include statistical signal processing, robust stochastic filtering, and game theory, with applications to positioning systems and wireless communications. He is the recipient of the 2014 EURASIP Best PhD Thesis Award, the 9th Duran Farell Award, and the 2016 ION Early Achievements Award.

\begin{abstract}
As Global Navigation Satellite Systems (GNSS) become increasingly important for the application of autonomous transportation, the characterization of the uncertainty present in GNSS signals has been a recurrent topic in the GNSS community. With multipath being the most pronounced source of error while navigating during challenging scenarios, characterizing this process is a growing requirement. Improper modeling of the measurements variances can reduce the accuracy of the estimation as much as a gross bias on these measurements. It is therefore required to have reliable and statistically meaningful modeling of errors. To isolate the multipath from the other error sources, the dual-frequency iono-corrected code-minus-carrier combination (CMC) is employed. Conjugate prior Bayesian analysis is used to characterize the variance of the multipath effect to later regress the unknown parameter of the proposed error models. For the derivation of these models, massive amounts of multi-frequency GPS and Galileo data was collected. The comparison between the proposed models was carried out with actual data from a challenging signal-degraded scenario, in terms of positioning and integrity monitoring performance.
\end{abstract}

Keywords - GNSS; Probabilistic Modeling; Multipath; Variance Model.

\section{INTRODUCTION}

GNSS technology has been under substantial development over the last years, especially with the evolution and operation of the European Unions Galileo and Chinas BeiDou constellations. From timing to weather prediction and location-based services, GNSS has become an integral part of everyday processes. With autonomous transportation being a major application of GNSS, the need for high accuracy positioning to ensure safe navigation is a necessity [1].

When considering autonomous transportation, it is critical to account for multipath, the most prominent source of error in terms of positioning while navigating during challenging scenarios [2]. This is especially the case in urban canyons and other elevated regions, where the high buildings, tree foliage and reflective structures result in reception Non-Line-of-Sight (NLOS) signals and multiple reflections (multipath). Another concerning factor surfaces when integrity monitoring is intended. Establishing inaccurate variance models of the GNSS measurements can result in reduced accuracy of the estimation and/or 
the complete failure of the integrity estimation. It is, therefore, a requirement to have reliable and statistically meaningful modeling of errors.

The characterization of the uncertainty present in GNSS signals has been a recurrent topic in the GNSS community. Thus, in [3], the first approach towards integrity monitoring was discussed, including a profound description on satellite weighting. This work was later extended in [4], where multi-frequency, multi-constellation systems were taken into consideration, consequently updating the GNSS-satellite variance models. Although much of these works play a crucial role for the aviation domain, its application to other areas has not yet been suggested, given the strong assumptions and the particular fault modes occurring in aviation. In [5], the question about how to monitor reliability and weight observations in complex scenarios was raised. For this case, data from a static scenario with degraded GNSS-signals was collected over a long time with the aim of formulating a new variance model based on signal strength. The usage of this model has lately become conventional for navigation in signal-degraded scenarios, as seen in [6]-[8]. In [9], multipath and receiver noise error were characterized using the combination of Code-Carrier and Divergence-Free smoothing, as a way to assess processing models for Ground Based Augmentation System. The regression of the parameters, however, was not validated with a real dynamic scenario to ensure reliable results.

This paper presents a methodology for the derivation of stochastic models for the characterization of multipath and receiver noise of GNSS code observables, based on Bayesian inference. First, the dual frequency code-minus-carrier combination is applied to determine the magnitude of multipath effects on GNSS code measurements. Then, based on conjugate prior Bayesian analysis, the variance of the multipath is expressed as inverse-Gamma distribution conditional on the elevation angle and/or the carrier-to-noise ratio of the observations. Finally, four multipath models are proposed, whose unknown parameters are learned from the prior inverse-Gamma distributions. The methodology is implemented using a massive amount of data collected on a dynamical scenario to provide multipath models for GPS L1, L2 and Galileo E1 and E5 frequencies. Moreover, this work includes the performance analysis of the proposed models using data from a signal-degraded scenario, where multipath effects severely compromise GNSS-dependent operations.

The rest of the paper is organized as follows. In section 2, the relevant mathematical methodology is presented. Section 3 describes the application of the methodology to learn the variance models. Section 4 refers to the performance validation of the aforementioned models on a challenging scenario. Finally, Section 5 presents the conclusions of the work, along with the next steps on the future work. Additionally, an appendix on the comparison of the multipath characterization of Galileo and GPS has been included.

\section{MeTHODOLOGY}

\section{A. Multipath Characterization}

The GNSS positioning problem is generally solved applying a Weighted Least Squares (WLS) adjustment. Classical Single Point Positioning (SPP) implies the use of pseudorange observations $\mathbf{y}$ to estimate the receiver coordinates and the receiver clock offset $\mathbf{x}=\left(x_{r}, y_{r}, z_{r}, d t_{r}\right)^{T}$ as

$$
\mathbf{x}=\arg \min _{\mathbf{x} \in \mathbb{R}^{m}}(\mathbf{y}-\mathbf{G x})^{T} \mathbf{W}(\mathbf{y}-\mathbf{G x})
$$

Here, $\mathbf{G} \in \mathbb{R}^{n \times m}$ contains the line-of-sight (LOS) unit direction vectors and the effects of the receiver clock offsets. W is the weight matrix, which is defined as $\mathbf{W}=\mathbf{Q}_{y y}^{-1}$, with $\mathbf{Q}_{y y}$ being the variance-covariance matrix of the observable:

$$
\mathbf{Q}_{y y}=\left[\begin{array}{ccc}
\sigma_{y_{1}}^{2} & & \\
& \ddots & \\
& & \sigma_{y_{n}}^{2}
\end{array}\right]
$$

where $\sigma_{y_{i}}^{2}$ comes from the uncertainty of the different error sources—satellite clocks, ephemeris, ionosphere, troposphere, multipath and receiver noise

$$
\sigma_{y_{i}}^{2}=\sigma_{c d t_{i}}^{2}+\sigma_{e p h_{i}}^{2}+\sigma_{I_{i}}^{2}+\sigma_{T r_{i}}^{2}+\sigma_{M_{i}}^{2}+\sigma_{\varepsilon_{i}}^{2}
$$

On signal-degraded environments, such as urban-canyons, multipath effects become the major source of pseudorange error. Therefore, multipath characterization becomes a critical task for properly weighing the satellites, and providing information to establish integrity monitoring. Prior to the explanation on multipath characterization, let us define the code and the carrier-phase observations:

$$
\begin{gathered}
R_{i}=\rho_{i}+c\left(d t_{r}-d t_{i}\right)+T r_{i}+I_{i}+\varepsilon_{i} \\
\Phi_{i}=\rho_{i}+c\left(d t_{r}-d t_{i}\right)+T r_{i}-I_{i}+\lambda N_{i}+\epsilon_{i}
\end{gathered}
$$


where

the subscript $i$ refers to the $i$-th satellite,

$R$ is the GNSS code observation,

$\Phi$ is the GNSS carrier phase observation (in meters),

$\rho$ is the geometric distance between the satellite and the unknown position of the receiver,

$c d t_{r}$ is the receiver clock offset delay, which without subscript refers to the satellite receiver,

$\operatorname{Tr}$ stands for the tropospheric effects,

$I$ gathers the ionospheric effects,

$\lambda$ is the carrier wavelength of the signal,

$N$ is the unknown carrier-phase ambiguity,

$\varepsilon$ and $\epsilon$ constitute the remaining errors (mainly multipath and receiver noise errors) in the code and carrier observations respectively.

Multipath effects are mostly considered a nuisance in GNSS measurements, so it is important to have an understanding of the magnitude of this error on the observations. Multipath combinations can be used for this purpose. The multi-frequency code-minus-carrier combination (CMC) is commonly applied for assessing pseudorange multipath [10]. Free of ionospheric and geometric effects, CMC clusters the remaining observables error terms: group-delay variations, carrier-phase ambiguities, receiver noise, and multipath.

$$
\begin{aligned}
\mathrm{CMC}_{i}^{f_{A}}= & R_{i}^{f_{A}}-\Phi_{i}^{f_{A}}+\frac{2}{\alpha}\left(\Phi_{i}^{f_{A}}-\Phi_{i}^{f_{B}}\right), \\
\mathrm{CMC}_{i}^{f_{A}}= & \varepsilon_{i}^{f_{A}}+\epsilon_{i}^{f_{A}}+\frac{2}{\alpha}\left(\epsilon_{i}^{f_{A}}+\epsilon_{i}^{f_{B}}\right)+ \\
& \underbrace{+\lambda^{f_{A}} N_{i}+\frac{2}{\alpha}\left(\lambda^{f_{A}} N_{i}-\lambda^{f_{B}} N_{i}\right)}_{\nu}
\end{aligned}
$$

where $\alpha=1-\left(f_{A} / f_{B}\right)^{2}$ and $\nu$ is a constant factor including carrier phase ambiguities and hardware delay biases [10]. Assuming that multipath and noise of the carrier-phase observations are negligible compared to the corresponding terms of the pseudorange and discarding $\nu$, which remains constant over time, the multipath and receiver noise term $\varepsilon$ for code observations is characterized as:

$$
\varepsilon^{f_{A}} \simeq \mathrm{CMC}^{f_{A}}-\nu
$$

\section{B. Probabilistic modeling of the variance}

Most of the existing models assume that the variance is an unknown deterministic constant. Alternatively, the variance can be considered as a random variable such that:

$$
\sigma^{2} \sim p\left(\sigma^{2} ; \theta, C / N_{0}\right)
$$

represents its probability density function, which depends on elevation and the carrier-to-noise ratio $C / N_{0}$ of the satellite. In conjugate prior Bayesian analysis, a popular choice for this distribution is the inverse-Gamma distribution. The inverseGamma has the desirable feature of having support only over the positive real values such that $\sigma^{2} \in \mathbb{R}_{+}$, which is very appropriate for variances.

Notice that since the distribution of $\sigma^{2}$ depends on the parameters $\left\{\theta, C / N_{0}\right\}$, its parameterization does also. Therefore, a complete probabilistic characterization would take that into account and write, more precisely, such that a model fitting needs to be done for each $\left\{\theta, C / N_{0}\right\}$ pair, with some granularity.

Consider a set of $N$ i.i.d. code noise samples $\varepsilon_{1: N} \triangleq\left\{\varepsilon_{1}, \ldots, \varepsilon_{N}\right\}$, obtained as in (9), for a given $\left\{\theta, C / N_{0}\right\}$ pair. A reasonable modeling of the error is:

$$
\varepsilon_{n} \sim \mathcal{N}\left(0, \sigma^{2}\left(\theta, C / N_{0}\right)\right)
$$

We are interested in the probabilistic representation of $\sigma^{2}\left(\theta, C / N_{0}\right)$. To infer such distribution, the conjugate Bayesian analysis was applied to estimate the variance of the sample $\varepsilon_{1: N}$ given a known mean [11].

The conjugate prior of the variances for the likelihood model represented in (11) is an inverse Gamma distribution

$$
p\left(\sigma^{2}\right)=\operatorname{IG}\left(\alpha_{0}, \beta_{0}\right)
$$



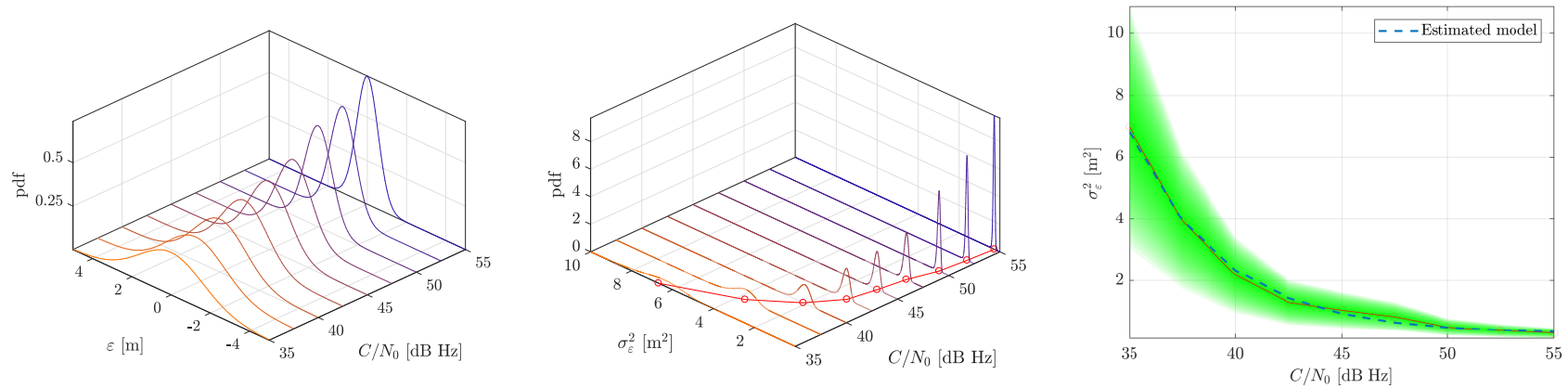

Fig. 1: Process flow example for the estimation of the $C / N_{0}$-based model. To the left, the estimated combination of the multipath and receiver noise is gridded for each $C / N_{0}$ division. In the middle, the $p d f$ for the inverse-Gamma distributions are shown, whose parameters are inferred from the previously gridded data. To the right, the mean of the IG distributions in red, the corresponding IG variances in green and in blue the model obtained after the regression.

with parameters $\alpha_{0}$ (shape) and $\beta_{0}$ (scale). For the sake of convenience, the dependence on the $\left\{\theta, C / N_{0}\right\}$ pair was dropped, but it goes without saying that each pair can have different parameters for (12). If no prior knowledge is available, a suitable objective prior results by setting $\alpha_{0}=0.001$ and $\beta_{0}=0.001$, such that the prior distribution is proper and relatively flat.

The prior is updated with the sample $\varepsilon_{1: N}$, yielding the posterior distribution

$$
p\left(\sigma^{2} \mid \varepsilon_{1: N}\right)=\operatorname{IG}\left(\alpha_{N}, \beta_{N}\right)
$$

where the parameters are computed as

$$
\begin{aligned}
& \alpha_{N}=\alpha_{0}+\frac{N}{2} \\
& \beta_{N}=\beta_{0}+\frac{1}{2} \sum_{n=1}^{N} \varepsilon_{n}^{2} .
\end{aligned}
$$

As a result, a distribution, (13), is obtained, which provides all the information available about the variance. In particular, the distribution informs about the uncertainty regarding the variance $\sigma^{2}$ for the given $\left\{\theta, C / N_{0}\right\}$ pair. Additionally, statistics can be computed from the posterior distribution (13) such as the mean:

$$
\operatorname{Mean}\left(\sigma^{2} \mid \varepsilon_{1: N}\right)=\frac{\beta_{N}}{\alpha_{N}-1}, \quad \text { for } \alpha_{N}>1
$$

the mode:

$$
\operatorname{Mode}\left(\sigma^{2} \mid \varepsilon_{1: N}\right)=\frac{\beta_{N}}{\alpha_{N}+1},
$$

and the variance of $\sigma^{2}$ given $\varepsilon_{1: N}$ :

$$
\operatorname{Var}\left(\sigma^{2} \mid \varepsilon_{1: N}\right)=\frac{\beta_{N}^{2}}{\left(\alpha_{N}-1\right)^{2}\left(\alpha_{N}-2\right)}, \quad \text { for } \alpha_{N}>2 .
$$

\section{Regression of variance means}

The topic of pseudorange stochastic modeling has been extensively studied by the GNSS community. For high precision applications, such as Real Time Kinematic (RTK), satellite elevation dependent models have often been applied [12]-[14]. For signal-degraded situations, the signal-to-noise ratio is considered a better quality indicator for GNSS observations. A model based on $C / N_{0}$ was first introduced in [5], and it has been widely adapted for navigating challenging scenarios [15]. This work compiles a collection of variance models for pseudorange weighting dependent on either satellite elevation angle, signal-to-noise ratio or the combination of both.

- Elevation model

$$
\sigma^{2}=a+\frac{b}{\sin \theta}
$$


- CNO-based model

$$
\sigma^{2}=a+b \cdot 10^{-C / N_{0} / 10}
$$

- Additive model

$$
\sigma^{2}=a+\frac{b}{\sin \theta}+c \cdot 10^{-C / N_{0} / 10}
$$

- Multiplicative model

$$
\sigma^{2}=a+b \cdot \frac{10^{-C / N_{0} / 10}}{\sin \theta}
$$

Finding the unknown parameters $a, b, c$ of these models constitute a nonlinear regression problem relating the mean of the estimated Inverse-Gamma distributions (16) and the corresponding $\left\{\theta, C / N_{0}\right\}$ pair, conditioned on the respective variance (18) for the distribution. Fig. 1 displays the flow for the proposed methodology. On the left, the estimated multipath error grid is shown according to the corresponding signal-to-noise ratio. The middle figures depicts the $p d f$ for the inverse-Gamma distribution of each $C / N_{0}$ division, whose parameters $\alpha$ and $\beta$ are inferred from the data corresponding to each $C / N_{0}$. On the right side, the estimated $C / N_{0}$-dependent variance model regression problem is shown.

\section{EXPERIMENTATION}

The methodology for deriving multipath models for pseudorange observations has been applied to actual data collected on board of the ferry "Mecklenburg-Vorpommern". As seen in Fig. 2, this vessel spans across the Baltic Sea between the ports of Rostock (Germany) and Trelleborg (Sweden) and is equipped with geodetic equipment, comprising a Javad DELTA receiver and a navXperience GNSS antenna. Unlike static data collection, where multipath represents a small share within the error budget, this scenario is profoundly affected by multipath from the high metallic structures of the ports and the high reflectivity of the salty water.

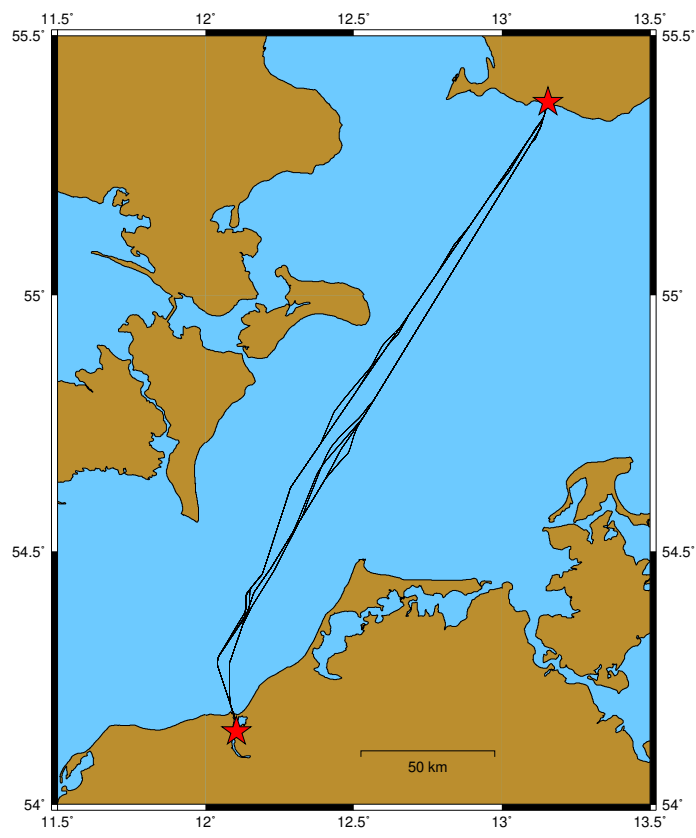

Fig. 2: Trajectory followed by the ferry connecting Rostock (Germany) and Trelleborg (Sweden), equipped with a geodetic receiver.

A total of three days of data was collected at $2 \mathrm{~Hz}$, from the 11th to 13th February 2018. The data collection includes the code and carrier-phase observations of GPS, Galileo and GLONASS satellites for different frequencies. Differently from GPS or Galileo, GLONASS employs the Frequency Division Multiple Access (FDMA) as a technique for their spectral separation [16]. While offering a higher protection against narrow-band jamming attacks, FDMA prevents the application of our proposed methodology for multipath modelling, as the multipath-free combination from Eq. 6 introduces additional errors [17]. Additional consideration was taken with regards to the effect of sparse outliers caused by extremely large multipath effects, as the assumption of zero-mean normal distributed data could be violated. The Bayesian inference of the multipath 
variance is sensitive to the aforementioned assumption and thus robust estimation theory is applied to bound the effect of the outliers. The Median Absolute Deviation (MAD) [18] was found for the observations corresponding to each pair of elevation and $C / N_{0}$ and only the data proportion within three MAD is preserved.

Table I gathers the estimated values for the unknown parameters of the proposed pseudorange models, see Eqs. 19 to 22. Further analysis on the experimentation, fit of the error models to the input data, and a comparison between GPS L1, L2 and Galileo E1, E5 can be found in the Appendix A.

TABLE I: Estimated values for the unknown parameters of the proposed multipath error models.

\begin{tabular}{|c|c|c|c|}
\hline Model & $\mathrm{a}$ & $\mathrm{b}$ & $\mathrm{c}$ \\
\hline \multicolumn{4}{|l|}{ GPS L1 } \\
\hline Elevation & 0.24 & 0.15 & - \\
\hline$C / N_{0}$-based & 0.75 & 3158.44 & - \\
\hline Additive & 0.37 & 0.24 & 2301.12 \\
\hline Multiplicative & 0.94 & 625.00 & - \\
\hline \multicolumn{4}{|l|}{ GPS L2 } \\
\hline Elevation & 0.31 & 0.20 & - \\
\hline$C / N_{0}$-based & 0.73 & 6042.09 & - \\
\hline Additive & 0.34 & 0.16 & 4797.93 \\
\hline Multiplicative & 0.68 & 884.02 & - \\
\hline \multicolumn{4}{|l|}{ Galileo E1 } \\
\hline Elevation & 0.19 & 0.19 & - \\
\hline$C / N_{0}$-based & 0.20 & 26224.18 & - \\
\hline Additive & 0.01 & 0.10 & 22730.32 \\
\hline Multiplicative & 0.45 & 3477.44 & - \\
\hline \multicolumn{4}{|l|}{ Galileo E5 } \\
\hline Elevation & 0.34 & 0.20 & . \\
\hline$C / N_{0}$-based & 0.67 & 8497.49 & - \\
\hline Additive & 0.31 & 0.17 & 6299.86 \\
\hline Multiplicative & 0.68 & 1234.73 & - \\
\hline
\end{tabular}

\section{VALIDATION}

The performance of the estimated multipath error models was evaluated using real observations recorded during the measurement campaign on 16th May 2017 (DOY 208, UTC 08:30-14:30) conducted in Koblenz (Germany) on the Moselle river, as seen in Fig. 3. The data was collected on board of the vessel MS Bingen at $1 \mathrm{~Hz}$, with an elevation mask of $5^{\circ}$. The equipment consisted of navXperience GNSS antennas connected to a geodetic Javad DELTA receiver. The ground truth reference corresponded to the multi-frequency GPS-only RTK solution from the RTKLIB open source software [19].

The comparison of the models comprises of an analysis of the positioning performance and the integrity monitoring of the navigation solution. Thus, a GPS L1 Single Point Positioning (SPP) solution is estimated, as well as the horizontal and vertical protection levels. Unfortunately, the largest positioning errors cannot be examined, as there is not a fixed RTK position for these epochs, mainly corresponding to bridge passings.

Table II gathers statistical indicators of the vertical positioning errors (VPE) for every model. Additionally, the first row presents the performance of the "Unweighted" model to exhibit the worsening of the positioning performance when no pseudorange variance model is applied. The root mean square (RMS) shows only subtle differences among the examined models, implying there is a low model influence on the performance during nominal situations. The major gain of the models occur under challenging situations with bigger positioning errors, as deduced from the $68 \%$ and $99.7 \%$ of the cumulative distribution function (CDF) of the errors. Signal-to-noise ratio becomes a more relevant indicator of the actual pseudorange quality than the satellite elevation, with an approximate improvement of $30 \%$ for the $99.7 \%$ VPE CDF between the elevation-based and the multiplicative models. There is also a noticeable closeness between the $C / N_{0}$-dependent models - $C / N_{0}$-based, additive and multiplicative. This indicates the weaker influence of the elevation angle compared to the signal-to-noise ratio, mainly due to the higher dominance (larger slope) of the exponential function over the sinusoidal function. 


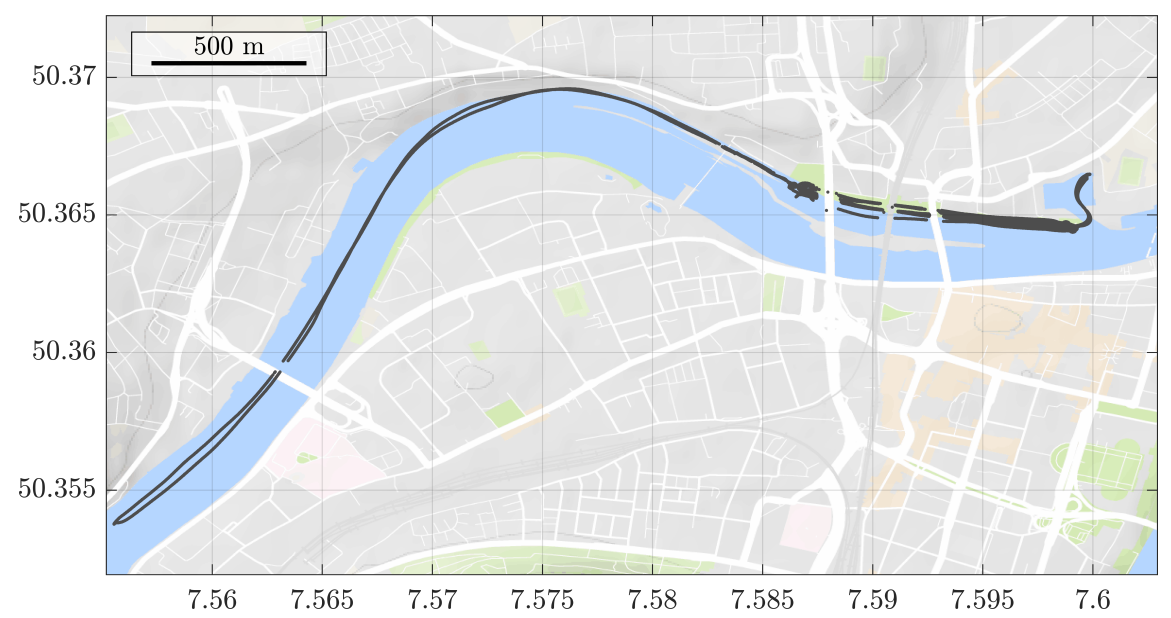

Fig. 3: Measurement area on the river Moselle near Koblenz (Germany), reference path marked in dark gray.

TABLE II: Comparison of the positioning performance for the error models evaluated.

\begin{tabular}{lrrc}
\hline & $\begin{array}{r}\text { RMS } \\
\text { VPE [m] }\end{array}$ & $\begin{array}{r}68 \% \text { CDF } \\
\text { VPE [m] }\end{array}$ & $\begin{array}{c}99.7 \% \text { CDF } \\
\text { VPE [m] }\end{array}$ \\
\hline Unweighted & 0.94 & 0.63 & 3.79 \\
\hline Elevation-based & $-\frac{-}{2}-86$ & 0.55 & 3.20 \\
$C / N_{0}$-based & 0.83 & 0.47 & 2.33 \\
Additive & 0.83 & 0.47 & 2.33 \\
Multiplicative & 0.80 & 0.46 & 2.26 \\
\hline
\end{tabular}

The second part of the model validation explores the performance of the models for integrity monitoring. Integrity is the measure of trust that can be placed in the correctness of the estimated navigation solution, and it has acquired a fundamental importance for the certified use of GNSS in aircraft navigation [20]. During regular operations, it is not possible to acknowledge the actual position error, so the protection level (PL) offers a statistical bound for the error of the estimated position. The main integrity requirement is that the horizontal or vertical positioning error must not be greater than the corresponding horizontal or vertical protection level (HPL, VPL) [21]. The Stanford Plot, first presented in [22], is a graphical tool to illustrate the integrity monitoring of a system. For each epoch, the estimated VPL is depicted against the corresponding VPE. The diagonal axis separates those samples, in which the position error is covered by the protection level (Normal Operation), from those below the diagonal, in which the protection level fails to cover the position error (Misleading operations) [23]. The Alert Limit (AL) is the error tolerance which might not be exceeded without issuing a warning, chosen for our study as 25 meters. The Stanford Plot distinguishes as hazardously misleading information (HMI) those operation faults in which the positioning error overpasses the AL without the corresponding PL alerting of such scenario.

Fig. 4 depicts the Stanford Plot for the four model analyzed. From Fig. 4(a), it is clear that the elevation-based model is the "tightest" protection levels, as only $3.6 \%$ of the solutions are labeled as unavailable. However, a concerning $0.2 \%$ of the epochs resulted in events for misleading operations, which means that the model is overoptimistic, as the protection levels were too low during these epochs. The rest of the models, highly influenced by the signal-strength of the observation, mostly eliminate the misleading operations at the expense of a lower unavailability. Only the additive model nullifies the number of failure epochs, although it has the lowest availability among the models. There is undoubtedly the challenge to find the proper balance between high availability and accuracy, while providing the proper measures for integrity monitoring.

\section{Summary AND OUTLOOK ON FUtURE Work}

This work presents a methodology to derive stochastic models for the characterization of multipath and receiver noise of GNSS code observables, based on Bayesian inference. The dual frequency code-minus-carrier combination provides a representation of the multipath free from the influence of ionospheric and geometric effects, and it is valid for all GNSS constellations whose spectral separation are based on CDMA techniques. Although several pseudorange variance models can be found in the GNSS-related literature, their estimation is mostly based on simple regression of the estimated error 




(a) Elevation-based model

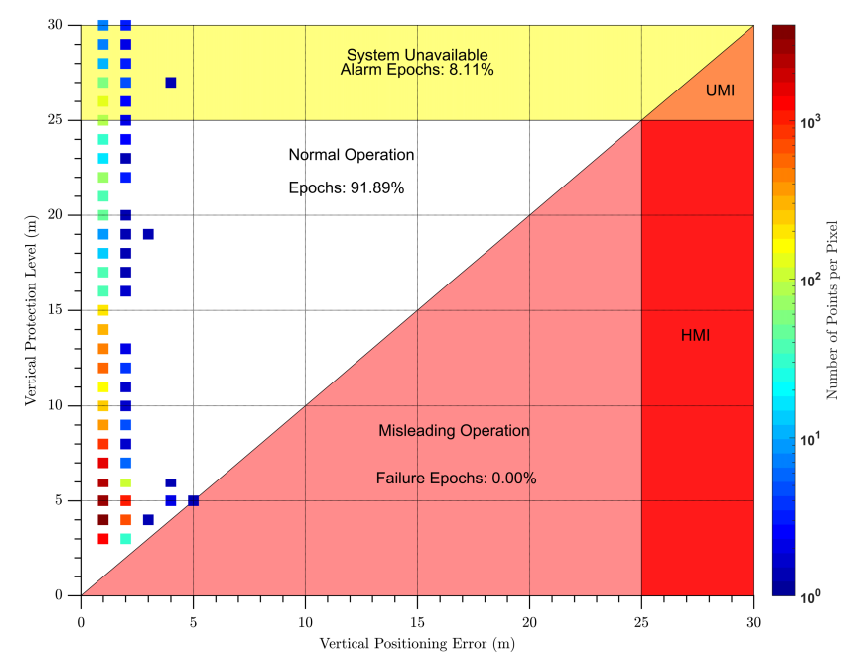

(c) Additive model

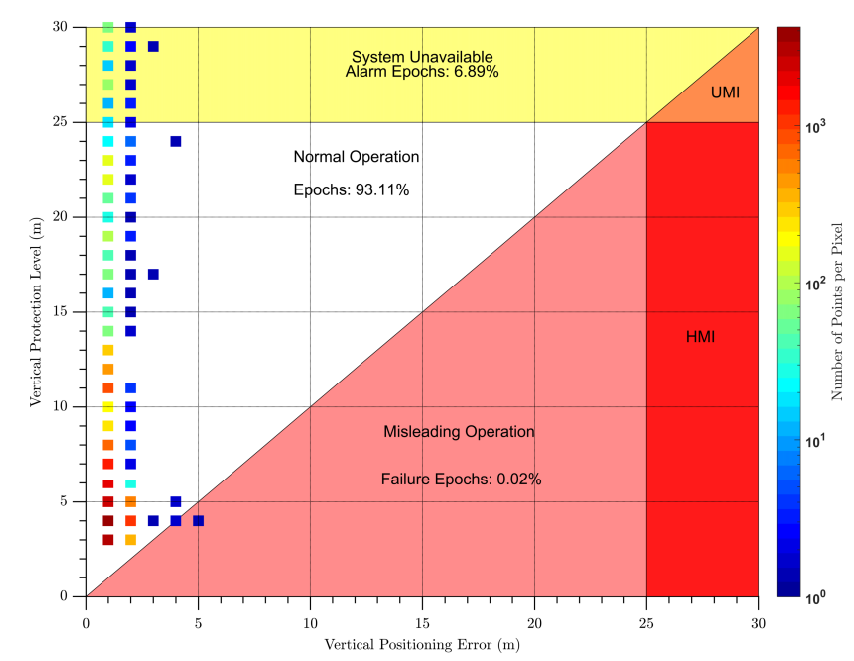

(b) $C / N_{0}$-based model

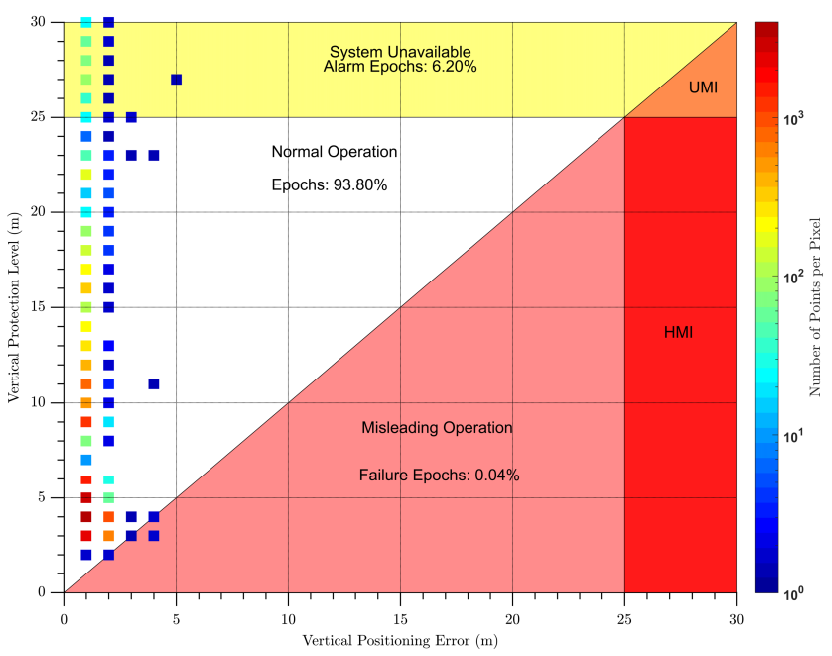

(d) Multiplicative model

Fig. 4: Stanford Plot depecting the integrity monitoring using the different multipath stochastic models presented.

budget calculated using data captured on a static scenario where the multipath influence is limited. This study comprises of the complete process of pseudorange variance model finding: first, the application of probabilistic modeling is introduced; then, a massive amount of data collected on a challenging scenario is used for the estimation of the unknown parameters of the models; finally, actual data of a different signal-degraded scenario is used for the validation of the models, analyzing the performance of the models in terms of positioning and integrity monitoring.

The framework of conjugate prior analysis applies perfectly to the problem of finding the weighting GNSS observations, as their noise is widely agreed on being zero-mean normally-distributed. The inverse-Gamma distribution results in the conjugate prior for the variance of the observations, and the parameters of the IG distributions estimated from the multipath combination. By determining the $p d f$ of the inverse-Gamma distributions of the GNSS noise for each pair of elevation and/or signal-to-noise ratio, it is possible to find the unknown parameters of the proposed error models. Following this procedure, not only are the variance models found but also the corresponding confidence bands. It is a challenging and prospective topic to include the uncertainty of the uncertainty into the problem of integrity monitoring, capturing the higher moments of ambiguity into the GNSS positioning problem. 


\section{APPENDIX A}

\section{COMPARISON GPS - GALILEO}

This appendix pertains to the differences between GPS and Galileo, devoting particular attention to the performance of Galileo E5 observations. Fig. 5 shows the satellite availability for both constellation on 11th February 2018 in the region of the Baltic Sea connecting Rostock (Germany) and Trelleborg (Germany). While Galileo availability still remains considerably lower than GPS (at the time of the publication of this paper, only 14 Galileo satellites are marked as usable [24]), a minimum of 4 Galileo satellites could be tracked for $78 \%$ of the time and the combined-constellation allows for the observation of at least 10 satellites for over $99.9 \%$ of a complete day. Note that Galileo satellites E14 and E18, having incorrect highly eccentric orbits, were discarded from our study - a detailed information on their use is recommended [25].

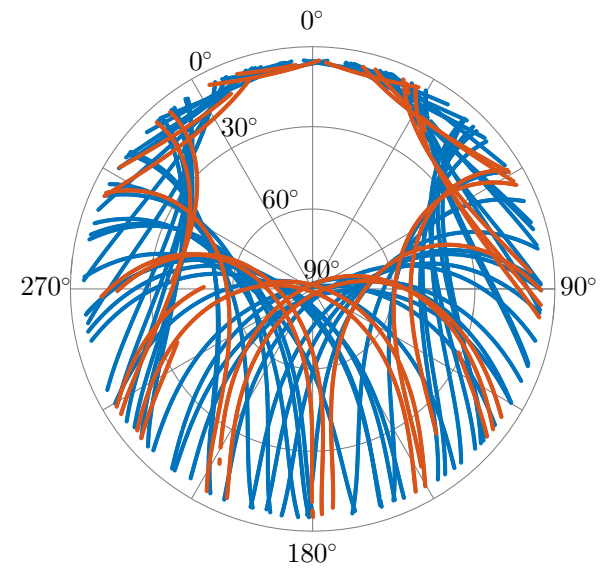

(a) Skyplot for the GPS and Galileo tracked satellites, in blue and orange respectively.

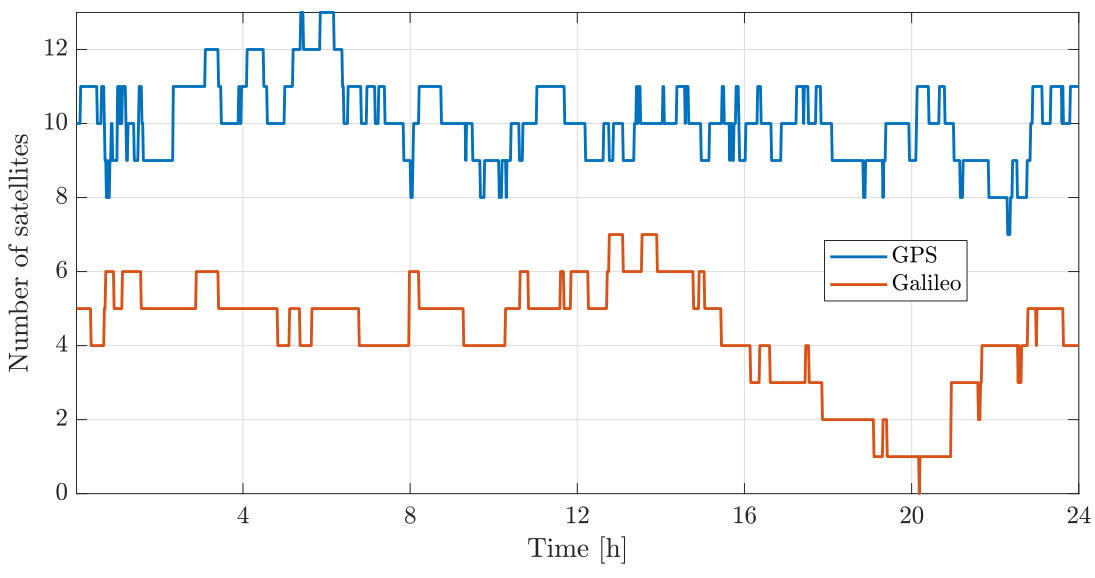

(b) Number of visible satellites over time.

Fig. 5: Availability of the tracked GPS, Galileo satellites on 11th February 2018 (DOY 042, UTC 00:00-23:59) in the area shown in Fig. 2.

Fig. 6 relates the carrier-to-noise ratio against the elevation angle of the GNSS observations, with the color implying the density of observations. As expected, the number of observations decrease with the satellite elevation and most of the measurements congregate between $5^{\circ}$ and $15^{\circ}$. Similar behavior is found for the carrier-to-noise ratio, as most of the observations clusters between 40 and $45 \mathrm{~dB} \mathrm{~Hz}$. The spread of the $C / N_{0}$ of for Galileo E5 is significantly more narrow than GPS L1 (GPS L1 $C / N_{0}$ standard deviation is $4.76 \mathrm{~dB} \mathrm{~Hz}$, Galileo E5 $C / N_{0}$ standard deviation is $3.85 \mathrm{~dB} \mathrm{~Hz}$ ).
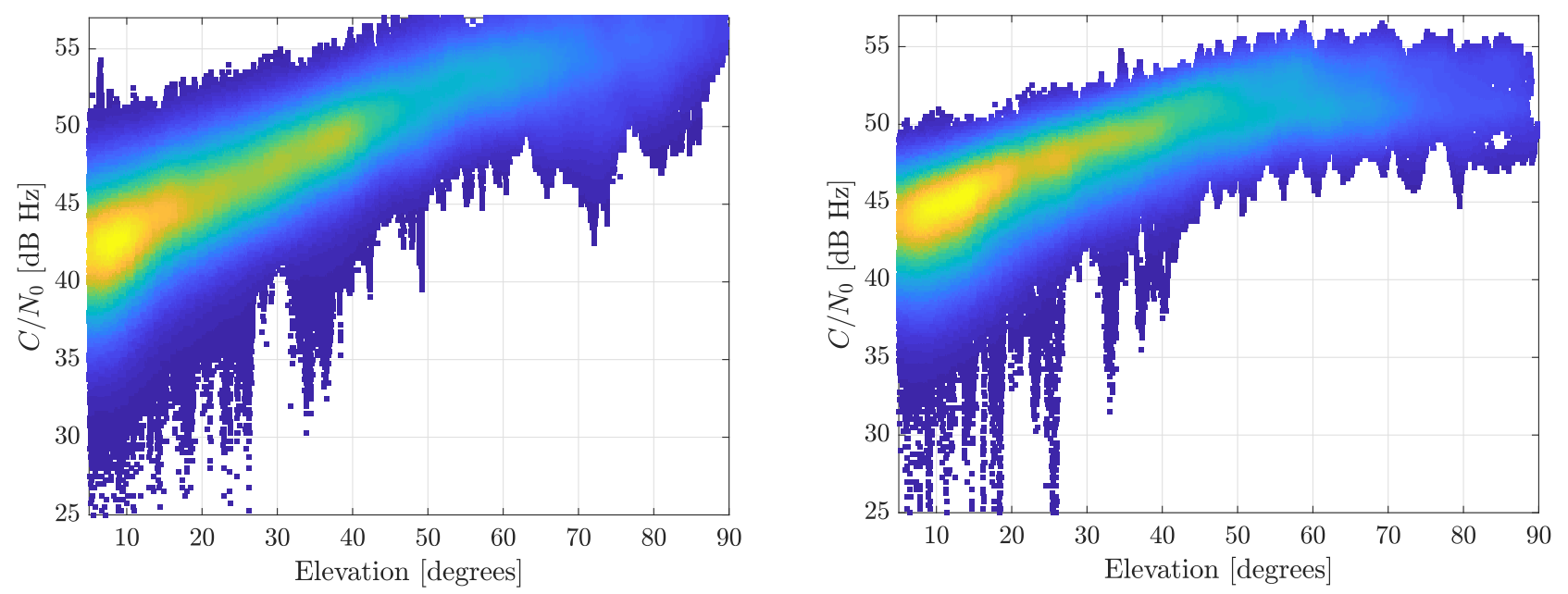

Fig. 6: 2D histogram plot of CN0 versus elevation angle for GPS L1 observations (left) and Galileo E5 (right).

Finally, it is compared the magnitude of the multipath affecting Galileo and GPS. Fig. 7 illustrates the first two moments for the IG distributions describing the multipath effects against the elevation angle, comparing GPS L1 with Galileo E5 
(in blue and orange respectively). As shown in previous studies [26], [27], Galileo E5 features a lower noise and a higher multipath suppression compared to GPS L1 frequency for satellites with elevation lower than $35^{\circ}$. Although Galileo highelevation satellites appear to be further affected by multipath, this phenomena is most likely due to the low volume of data of such characteristics.

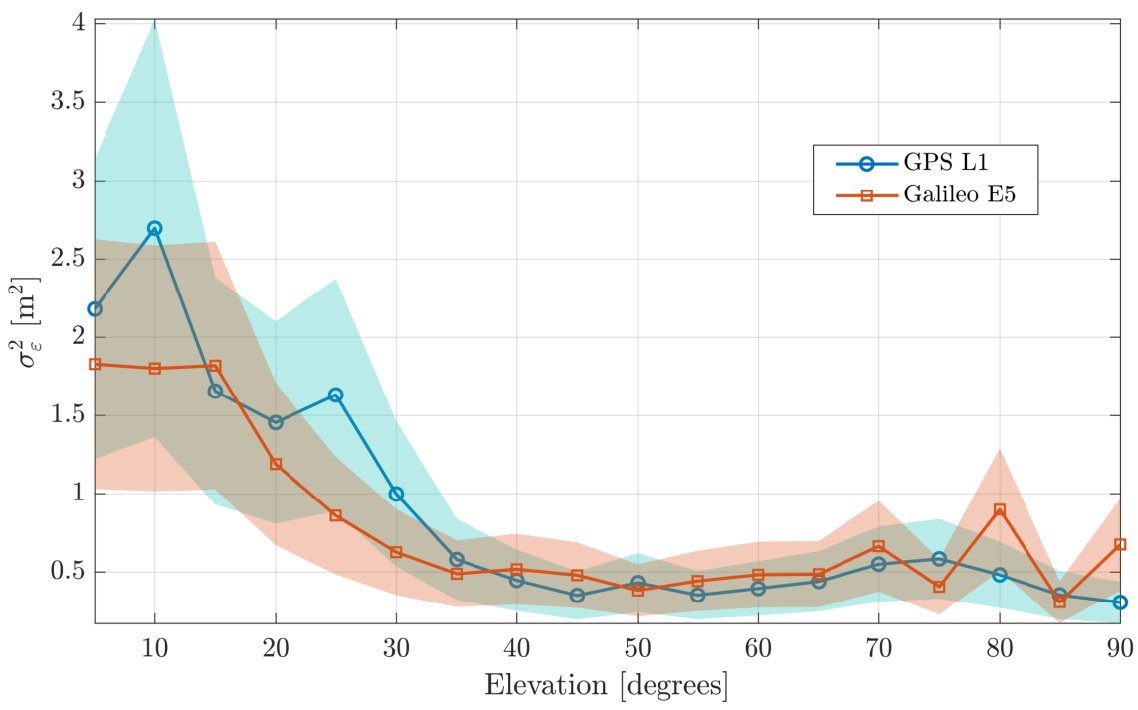

Fig. 7: Mean of the inverse-Gamma distribution representing the multipath variance for each elevation angle division.

\section{ACKNOWLEDGMENTS}

We, the authors, would like to thank the partners from the LAESSI project for their help during the measurement campaign in Koblenz 2017, as well as the crews from the Mecklenburg-Vorpommern and the MS Bingen vessels for their collaboration. Also, we greatly appreciate the priceless help and cartography skills offered by Iván Herrera Pinzón.

\section{REFERENCES}

[1] N. Williams, G. Wu, and P. Closas, "Impact of positioning uncertainty on eco-approach and departure of connected and automated vehicles," in Position, Location and Navigation Symposium (PLANS), 2018 IEEE/ION. IEEE, 2018, pp. 1081-1087.

[2] M. G. Amin, P. Closas, A. Broumandan, and J. L. Volakis, "Vulnerabilities, threats, and authentication in satellite-based navigation systems [scanning the issue]," Proceedings of the IEEE, vol. 104, no. 6, pp. 1169-1173, 2016.

[3] T. Walter and P. Enge, "Weighted RAIM for precision approach," in PROCEEDINGS OF ION GPS, vol. $8 . \quad$ Institute of Navigation, 1995, pp. 1995-2004.

[4] J. Blanch, T. Walter, P. Enge, Y. Lee, B. Pervan, M. Rippl, and A. Spletter, "Advanced RAIM user algorithm description: Integrity support message processing, fault detection, exclusion, and protection level calculation," in Proceedings of the 25th International Technical Meeting of The Satellite Division of the Institute of Navigation (ION GNSS 2012), 2012, pp. 2828-2849.

[5] H. Kuusniemi, A. Wieser, G. Lachapelle, and J. Takala, "User-level reliability monitoring in urban personal satellite-navigation," IEEE Transactions on Aerospace and Electronic Systems, vol. 43, no. 4, 2007.

[6] Z. Jiang and P. D. Groves, "GNSS NLOS and multipath error mitigation using advanced multi-constellation consistency checking with height aiding," 2012.

[7] D. Medina, M. Romanovas, I. Herrera-Pinzón, and R. Ziebold, "Robust position and velocity estimation methods in integrated navigation systems for inland water applications," in Position, Location and Navigation Symposium (PLANS), 2016 IEEE/ION. IEEE, 2016, pp. 491-501.

[8] S. Gaglione, A. Innac, S. P. Carbone, S. Troisi, and A. Angrisano, "Robust estimation methods applied to GPS in harsh environments," in Navigation Conference (ENC), 2017 European. IEEE, 2017, pp. 14-25.

[9] G. Rotondo, P. Thevenon, C. Milner, C. Macabiau, M. Felux, A. Hornbostel, and M. S. Circiu, "Methodology for determining Pseudorange noise and multipath models for a multi-constellation, multi-frequency GBAS system," in International Technical Meeting $2015,2015$.

[10] P. Teunissen and O. Montenbruck, Springer handbook of global navigation satellite systems. Springer, 2017.

[11] J. M. Bernardo and A. F. M. Smith, Bayesian Theory. Wiley \& sons, 1994.

[12] C. Eling, L. Klingbeil, and H. Kuhlmann, "Real-time single-frequency GPS/MEMS-IMU attitude determination of lightweight UAVs," Sensors, vol. 15, no. 10, pp. 26212-26235, 2015.

[13] B. Li, "Stochastic modeling of triple-frequency BeiDou signals: estimation, assessment and impact analysis," Journal of Geodesy, vol. 90, no. 7, pp. 593-610, 2016.

[14] R. Odolinski and P. J. Teunissen, "Low-cost, high-precision, single-frequency GPS-BDS RTK positioning," Gps Solutions, vol. 21, no. 3, pp. 1315$1330,2017$.

[15] A. Angrisano, C. Gioia, S. Gaglione, and G. Del Core, "GNSS reliability testing in signal-degraded scenario," International Journal of Navigation and Observation, vol. 2013, 2013.

[16] J. S. Subirana, M. Hernandez-Pajares, and J. e Miguel Juan Zornoza, GNSS Data Processing: Fundamentals and Algorithms. European Space Agency, 2013. 
[17] J. Wang, C. Rizos, M. P. Stewart, and A. Leick, "GPS and GLONASS integration: modeling and ambiguity resolution issues," GPS solutions, vol. 5, no. 1, pp. 55-64, 2001

[18] P. J. Rousseeuw and C. Croux, "Alternatives to the median absolute deviation," Journal of the American Statistical association, vol. 88, no. 424, pp. 1273-1283, 1993.

[19] T. Takasu and A. Yasuda, "Development of the low-cost RTK-GPS receiver with an open source program package RTKLIB," in International symposium on GPS/GNSS. International Convention Center Jeju Korea, 2009, pp. 4-6.

[20] T. Walter, P. Enge, J. Blanch, and B. Pervan, "Worldwide vertical guidance of aircraft based on modernized GPS and new integrity augmentations," Proceedings of the IEEE, vol. 96, no. 12, pp. 1918-1935, 2008.

[21] P. Enge, T. Walter, S. Pullen, C. Kee, Y.-C. Chao, and Y.-J. Tsai, "Wide area augmentation of the Global Positioning System," Proceedings of the IEEE, vol. 84, no. 8, pp. 1063-1088, Aug 1996.

[22] A. Hansen, "WAAS Precision Approach Metrics: Accuracy, Integrity, Continuity and Availability," URL: http://waas. stanford. edu/metrics. html [accessed 1 November 2004], 2004.

[23] M. Tossaint, J. Samson, F. Toran, J. Venture-Traveset, M. Hernandez-Pajares, J. Juan, J. Sanz, and P. Ramos-Bosch, "The Stanford-ESA Integrity Diagram: A New Tool for The User Domain SBAS Integrity Assessment," Navigation, vol. 54, no. 2, pp. 153-162, 2007.

[24] E. G. N. satellite Systems Agency. (2018) Galileo constellation information. [Online]. Available: https://www.gsc-europa.eu/systemstatus/Constellation-Information

[25] J. Paziewski, R. Sieradzki, and P. Wielgosz, "On the Applicability of Galileo FOC Satellites with Incorrect Highly Eccentric Orbits: An Evaluation of Instantaneous Medium-Range Positioning," Remote Sensing, vol. 10, no. 2, p. 208, 2018.

[26] A. Simsky, D. Mertens, J.-M. Sleewaegen, M. Hollreiser, and M. Crisci, "Experimental results for the multipath performance of galileo signals transmitted by GIOVE-A satellite," International Journal of Navigation and Observation, vol. 2008, 2008.

[27] H. T. Diessongo, H. Bock, T. Schüler, S. Junker, and A. Kiroe, "Exploiting the galileo E5 wideband signal," Inside GNSS, vol. 7, no. 5, pp. 64-73, 2012. 